\title{
Early Odorant Exposure Increases the Number of Mitral and Tufted Cells Associated with a Single Glomerulus
}

\author{
(ㄱ)Annie Liu, ${ }^{1,2,3}$-Sajishnu Savya, ${ }^{1}$ and ${ }^{-N a t h a n i e l ~ N . ~ U r b a n ~}{ }^{1,2,3,4}$ \\ ${ }^{1}$ Center for Neuroscience at the University of Pittsburgh and ${ }^{2}$ Department of Neurobiology, University of Pittsburgh, Pittsburgh, Pennsylvania 15213, \\ ${ }^{3}$ Center for the Neural Basis of Cognition, Pittsburgh, Pennsylvania 15213, and ${ }^{4}$ Department of Biological Sciences, Carnegie Mellon University, Pittsburgh, \\ Pennsylvania 15213
}

The highly specific organization of the olfactory bulb $(\mathrm{OB})$ is well known, but the impact of early odorant experience on its circuit structure is unclear. Olfactory sensory neurons (OSNs) project axons from the olfactory epithelium to the OB, where they form spherical neuropil structures called glomeruli. These glomeruli and the postsynaptic targets of OSNs, including mitral and tufted cells (M/TCs) and juxtaglomerular cells, form glomerular modules, which represent the basic odor-coding units of the OB. Here, we labeled M/TCs within a single glomerular module of the mouse $\mathrm{OB}$ and show that odorant exposure that starts prenatally and continues through postnatal day 25 has a major impact on the structure of the glomerular module. We confirm that exposure increases the volume of the activated glomeruli and show that exposure increases M/TC number by $>40 \%$ in a glomerulus-specific fashion. Given the role of M/TCs in $\mathrm{OB}$ output and in lateral inhibition, increasing the number of M/TCs connected to a single glomerulus may also increase the influence of that glomerulus on the $\mathrm{OB}$ network and on $\mathrm{OB}$ output. Our results show that early odorant exposure has a profound effect on $\mathrm{OB}$ connectivity and thus may affect odorant processing significantly.

Key words: glomerulus; mitral cells; olfaction; plasticity

\section{Significance Statement}

Experience shapes neural circuits in a variety of ways, most commonly by changing the strength of activated connections. Relatively little is known about how experience changes circuitry in the olfactory system. Here, we show that for a genetically identified glomerulus in the mouse olfactory bulb, early odorant exposure increases the number of associated mitral and tufted cells by $40 \%$ and $100 \%$, respectively. Understanding the structural changes induced by early odorant experience can provide insight into how bulbar organization gives rise to efficient processing. We find that odorant experience increases the number of projection neurons associated with a single glomerulus significantly, a dramatic and long-lasting structural change that may have important functional implications.

\section{Introduction}

The structure of the mouse olfactory bulb (OB) demonstrates remarkable regularity from animal to animal. Olfactory sensory neurons (OSNs) expressing the same odorant receptor (OR) co-

\footnotetext{
Received Feb. 28, 2016; revised Aug. 17, 2016; accepted Sept. 7, 2016.

Author contributions: A.L. and N.N.U. designed research; A.L. performed research; A.L. contributed unpublished reagents/analytic tools; A.L. and S.S. analyzed data; A.L., S.S., and N.N.U. wrote the paper.

This work was supported by National Institute on Deafness and Other Communication Disorders-National Institutes of Health (Grant R01DC011184 to N.N.U. and Grant F30DC015161 to A.L.) and the Pennsylvania Department of Health Commonwealth Universal Research Enhancement Program (N.N.U.). We thank Shawn Burton, Matthew Geramita, and Claire Cheetham for helpful comments; Greg LaRocca for technical help with tissue processing; and Diego Restrepo and Jo Todrank for helpful comments and technical advice regarding odorant exposure and behavioral analysis.

The authors declare no competing financial interests.

Correspondence should be addressed to Nathaniel N. Urban, Department of Neurobiology, University of Pittsburgh, E1440 BSTWR, 200 Lothrop Street, Pittsburgh, PA 15213. E-mail: nurban@pitt.edu.

DOI:10.1523/JNEUROSCI.0654-16.2016

Copyright $\odot 2016$ the authors $\quad 0270-6474 / 16 / 3611646-08 \$ 15.00 / 0$
}

alesce into $\sim$ two glomeruli per $\mathrm{OB}$, with relatively low positional variance across animals, the amplitude of which is related to OR identity (Mombaerts et al., 1996; Strotmann et al., 2000; Schaefer et al., 2001; Feinstein and Mombaerts, 2004; Zapiec and Mombaerts, 2015). Although location is determined by OR identity, odorant conditioning increases the rate of coalescence and the precision of axonal targeting (Kerr and Belluscio, 2006; Dias and Ressler, 2014), suggesting that both genetic and experiencedependent mechanisms govern the development of reliable OB glomerular patterns. However, the role of experiencedependent mechanisms in shaping other components of olfactory circuitry is unknown.

OSN axons reach a targeted location in the OB by approximately embryonic day (E) 15, and the formation of early glomerular structures is observed by E16-E20 (Royal and Key, 1999; Blanchart et al., 2006), but other glomeruli may form in early postnatal days (P) (Potter et al., 2001). Mitral cell (MC) apical 
dendrites infiltrate the glomerular layer by E17, with some neurons initially sending apical dendrites to multiple glomeruli (Blanchart et al., 2006). Pruning of these apical dendrites occurs by $\mathrm{P} 10$, at which point each cell has one branched apical dendrite receiving OSN input in a single glomerulus (Malun and Brunjes, 1996; Matsutani and Yamamoto, 2000; Blanchart et al., 2006) and may be influenced by activity (Lin et al., 2000). Therefore, the assignment of mitral and tufted cells (M/TCs) to specific glomerular modules is determined early in development through a pruning process. Figure $1 D$ depicts a very basic schematic of multiple glomeruli and a few of their postsynaptic targets, including periglomerular cells located in the glomerular layer, TCs in the external plexiform layer (EPL), and MCs in the mitral cell layer (MCL). Both OR identity and activation play major roles in glomerular development (Wang et al., 1998); prenatal and early postnatal odorant exposure increases the volume of activated glomeruli, increases the incidence of supernumerary glomeruli, and biases odorant preference toward the exposed ligand (Todrank et al., 2011; Valle-Leija et al., 2012). As a result, early odorant exposure may also influence the development of other OB neurons, such as by increasing the number of projection neurons connected to activated glomeruli.

Whether the same glomerular module recruits a specific number of M/TCs across animals consistently has not been explored, but may be an important feature for odor coding. The number of M/TCs associated with a specific glomerulus could affect the coding capacity of glomerular modules and alter the functional relevance of particular glomeruli. Glomerular modules with more M/TCs may exert more lateral inhibition (Egger and Urban, 2006) and provide more output to olfactory cortical areas. Understanding the influence of experience on M/TC number and glomerular module composition will provide insight into how experience affects odorant representation in the OB.

Here, we used in vivo electroporation to label and quantify the M/TCs connected to the M72 glomerulus (M72 M/TCs). We used the M72-IRES-tauGFP mouse line, in which M72-ORexpressing OSNs also express GFP, allowing for the targeting of a single genetically identified glomerulus across animals (Potter et al., 2001). After prenatal and early postnatal odorant exposure with methyl salicylate, a strong M72 ligand (Zhang et al., 2012), we analyzed M72 M/TC number and location, as well as glomerulus volume as defined by the spread of M72-expressing OSN axons and food preference as measured by sniffing time. We show that, in addition to increasing the size of the M72 glomerular module and the preference for methyl-salicylate-scented food, the number of M72 M/TCs can be increased by prenatal and early postnatal methyl salicylate exposure.

\section{Materials and Methods}

Subjects. Homozygote male and female M72-IRES-tauGFP mice were used for the majority of experiments except for one control behavior experiment, in which heterozygote male and female M72-IRES-ChR2YFP mice were used. Control and methyl-salicylate-exposed mice completed the methyl-salicylate-scented versus control food behavior task at P24 and were killed at P25 for anatomical studies. Two additional groups of mice (control and odor-exposed) also completed the methylsalicylate-scented versus control food behavior task between P24 and P26 and were used for in vivo electroporation studies from P25 to P40. There was no effect of age or sex on these measured parameters, so data were pooled. A third cohort of animals was exposed to hexanal-scented food, completed the hexanal-scented versus control food behavior task at P24, and was killed at P25-P35 for anatomical studies. A fourth cohort of M72-ChR2-YFP mice that had not undergone odorant exposure com- pleted the hexanal-scented versus control food behavior task between P26 and P36.

Odorant exposure. Prenatal and postnatal odorant exposure were performed on M72-IRES-tauGFP mice as described previously (Todrank et al., 2011). Briefly, food was mixed with either methyl salicylate or hexanal ( $1 \%$ by volume) and dried for $3 \mathrm{~d}$ in a glass dish under a fume hood. Breeding pairs were fed exclusively with scented food immediately after establishment and after litter birth throughout nursing until litters were weaned at P22-P24. Odorant-exposed litters were fed continuously with methyl-salicylate- or hexanal-odorized food until they were killed for anatomical studies at P25-P36 or had their OBs electroporated at P25$\mathrm{P} 40$. Mice were weighed for the first $3 \mathrm{~d}$ of odorant exposure to ensure normal growth while on the scented food.

In vivo electroporation. The in vivo electroporation procedure was adapted from the procedures detailed previously (Nagayama et al., 2007, Hovis et al., 2010, and He et al., 2012). Briefly, P25-P40 mice were anesthetized using a ketamine/xylazine intraperitoneal injection and a craniotomy $0.75 \mathrm{~mm}$ in diameter was made above each OB. Target glomeruli were visualized using two-photon microscopy. Monopolar glass electrodes (5-7 M $\Omega$ resistance) were back-filled with dye-containing solutions composed of Alexa Fluor 594 hydrazide or Alexa Fluor 594 dextran in PBS (for theta-glass double-label experiments, the dyes used were Alexa Fluor 594 dextran and Alexa Fluor 488 dextran in PBS and electrodes were pulled using theta capillary glass). After electrode placement in the center of the target glomerulus, 150 current pulses were delivered ( $0.01 \mathrm{~mA}$ in amplitude, $500 \mathrm{~ms}$ in duration, interpulse interval of $1.5 \mathrm{~s})$. After electroporation, mice were killed using cardiac perfusion with cold $4 \%$ paraformaldehyde. Criteria for successful electroporation were as follows: (1) glomerulus boundaries as defined by GFP-expressing OSN axons were completely filled by dye, (2) the perimeter of labeled juxtaglomerular cells (JGCs) surrounded the targeted glomerulus, and (3) apical dendrites of filled $\mathrm{M} / \mathrm{T}$ cells could be followed clearly back to the targeted glomerulus.

Tissue processing. After fixation, tissue was cryoprotected (30\% sucrose solution) and cut into $100-\mu \mathrm{m}$-thick sections. Targeting of GFP glomeruli was performed under two-photon imaging in vivo, but confocal imaging was performed on sectioned, fixed $\mathrm{OB}$ tissue to enable the clearest identification of M/TC apical dendrites targeting the M72 glomerulus. $Z$-stacks ( $2 \mu \mathrm{m}$ optical sections) were obtained for each tissue section using a Zeiss LSM 510 Meta DuoScan Spectral Confocal Microscope. Image analysis and measurements were performed in FIJI (RRID: SCR_002285) and Matlab (RRID: SCR_001622).

Food preference assessment. Mice were tested for food preference as described previously (Todrank et al., 2011) after P24. Mice were placed into a 38.1-cm-diameter circular arena with two different food pellets placed into wire mesh containers at opposite sides of the arena. Behavior was recorded for 3 min starting from time mouse began exploring the arena. Food preference was assessed by comparing time spent sniffing a scented food pellet versus a control food pellet. Mice were excluded from analysis if they climbed on top of and became entangled with the wire mesh containers $(n=3)$.

M/TC identification. After electroporation, the number of M72 M/TCs was determined through the $z$-stack of each OB section, avoiding double counting of cell bodies that span physical sections (data are presented as mean $\pm \mathrm{SD}, p$-values were determined by unpaired $t$ test). Labeled cells with cell somata larger than $>10 \mu \mathrm{m}$ and located $>50 \%$ within the MCL were counted as MCs; labeled cells with cell somata larger than $>10 \mu \mathrm{m}$ and located within the EPL were counted as TCs. External TCs were not analyzed. The locations of M/TCs were mapped on a coordinate system determined by anatomical landmarks (the M72 glomerulus center and the MCL below the glomerulus) to create a glomerulus-centric 3D map of M/TC spatial distribution that could be compared across animals. For each cell, we measured: A, the absolute distance from the center of the soma to the glomerulus center, and B, the angle between the perpendicular line from the MCL to the glomerulus center and the line formed by the glomerulus center and the soma center. $x$ and $y$ Cartesian coordinates were obtained by the following equations: $x=-\mathrm{A} * \sin (\mathrm{B})$ and $y=\mathrm{A} *$ $\cos (\mathrm{B})$. The $z$ cartesian coordinate was the distance of the cell from the optical section that contained the maximum glomerular diameter. The 
A

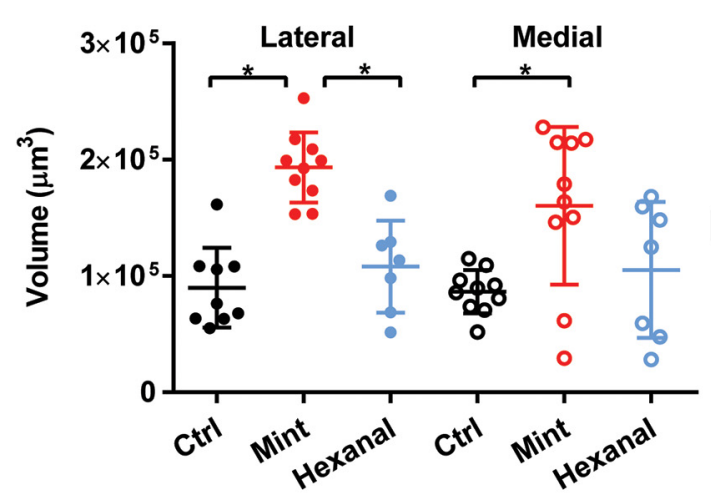

B Control

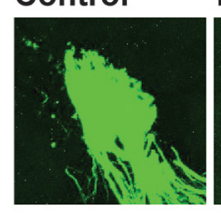

Mint

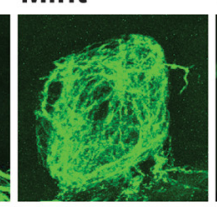

Hexanal

D

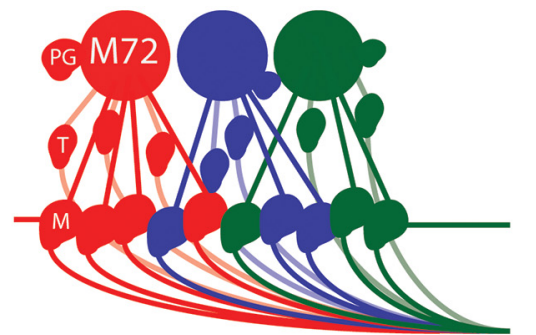

C

Time Spent Sniffing Scented Food

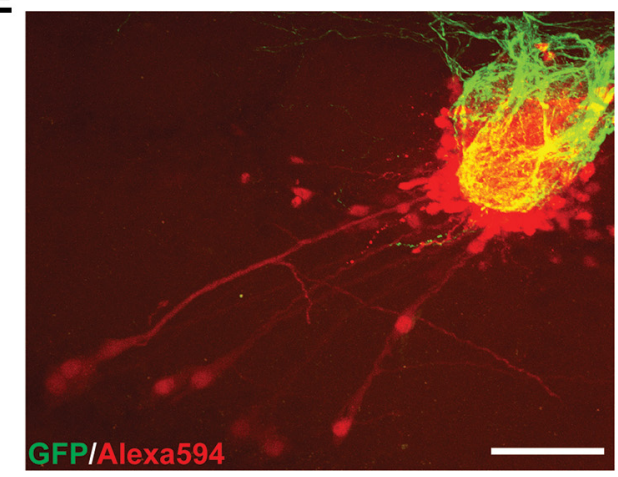

Figure 1. Prenatal and early postnatal methyl salicylate exposure increases $M 72$ glomerulus volume and changes food preference. $A$, Prenatal and early postnatal odorant exposure using food odorized with methyl salicylate (mint, $1 \%$ by volume) increases the size of the lateral and medial M72 glomeruli, but food odorized with hexanal (hexanal, $1 \%$ by volume) has no effect. ${ }^{*} p<0.01$. $\boldsymbol{B}$, Maximum projection images of example glomeruli of control and odor-exposed (mint and hexanal) animals at P25-P35. Scale bar, $50 \mu \mathrm{m}$. C, Prenatal and early postnatal odorant exposure using food odorized with methyl salicylate (mint, $1 \%$ by volume) or hexanal ( $1 \%$ by volume) increases the ratio of time spent sniffing scented food to total time spent sniffing food. Key: Control $\mid \mathrm{M}$, Control mice in preference test between methyl-salicylate-scented and control food; Mint $\mid \mathrm{M}$, methyl-salicylate-exposed animals in preference test between methyl-salicylate-scented and control food; Control $\mid \mathrm{H}$, control mice in preference test between hexanal-scented and control food; Hexanal $\mid \mathrm{H}$, hexanal-exposed animals in preference test between hexanal-scented and control food. ${ }^{*} p<$ 0.05. D, Schematic of three glomeruli with basic postsynaptic targets comprising three glomerular modules. Example M72 glomerulus is labeled, along with periglomerular cells (PG), TCs (T), and mitral cells (M). E, Example maximum projection image of electroporation-labeled M72 glomerulus (control) showing GFP-expressing OSN axons coalescing into a single glomerulus and Alexa Fluor 594 hydrazide-filled JGCs and M/TCs. Scale bar, $50 \mu \mathrm{m}$.

distance from the MCL to the glomerular layer was used to normalize measurements between physical sections and animals. Glomerular volume was measured by tracing the outline of the GFP-expressing axons in the glomerular layer within each optical Z-stack section.

\section{Results}

Early methyl salicylate exposure increases M72 glomerulus volume and changes food preference

When mice were fed food odorized with methyl salicylate (mint), a strong M72 ligand, the size of the M72 glomerulus increased in M72-IRES-GFP mice (Fig. 1). This confirms previous work showing that similar exposure to isopropyl tiglate, another strong M72 ligand with a mint-like smell, also increases M72 glomerulus volume (Todrank et al., 2011). We used methyl salicylate because it activates M72 more selectively, at least compared with some other nearby glomeruli (Zhang et al., 2012). Methyl salicylate exposure (from E0 to P25) increased the volume of both the medial and lateral glomeruli (Fig. 1 A,B; one-way ANOVA with Tukey's multiple-comparisons test; Control vs Mint; Mean \pm SD; lateral $0.89 \pm 0.34$ vs $1.93 \pm 0.3010^{5} \mu \mathrm{m}^{3}, n=9$ vs 10 , adjusted $p<0.0001$; medial $0.86 \pm 0.19$ vs $1.60 \pm 0.6810^{5} \mu \mathrm{m}^{3}$, $n=10$ vs 10 , adjusted $p=0.0062)$. At P24-P26, these same odor-exposed mice showed a preference for mint-scented food as opposed to control food (Fig. 1C; Kruskal-Wallis test with
Dunn's multiple-comparisons test; Control | M vs Mint | M; mean $\pm \mathrm{SD}$ of ratio of time spent sniffing mint-scented food to total time spent sniffing food: $0.39 \pm 0.15$ vs $0.57 \pm 0.09, n=15$ vs 22 , adjusted $p=0.0170$ ).

To examine the specificity of these anatomical and behavioral changes, we performed food-based odorant-exposure on a third cohort of animals using hexanal, which is known to not activate M72 receptors. Prenatal and early postnatal hexanal exposure did not increase the volume of either the medial or lateral glomeruli compared with control animals (Fig. $1 A, B$; one-way ANOVA with Tukey's multiple-comparisons test; control vs hexanal; mean $\pm \mathrm{SD}$; lateral $0.89 \pm 0.34$ vs $1.08 \pm 0.3910^{5} \mu \mathrm{m}^{3}, n=9$ vs 7 , adjusted $p=$ 0.9633 , n.s.; medial $0.86 \pm 0.19$ vs $1.05 \pm 0.5810^{5} \mu \mathrm{m}^{3}, n=10$ vs 7 , adjusted $p=0.9544$, n.s.). Comparing mint- and hexanal-exposed glomeruli revealed a significant difference between the volumes of mint-exposed and hexanal-exposed glomeruli (one-way ANOVA with Tukey's multiple-comparisons test, adjusted $p=0.0038$ ), but not between mint-exposed and hexanal-exposed medial glomeruli (one-way ANOVA with Tukey's multiple-comparisons test, adjusted $p=0.1337$, n.s.). Hexanal-exposed animals also showed a preference for hexanal-exposed food as opposed to control food (Fig. 1C; Kruskal-Wallis test with Dunn's multiple-comparisons test; Control $\mid \mathrm{H}$ vs Hexanal $\mid \mathrm{H}$; mean $\pm \mathrm{SD}$ of ratio of time spent 
A
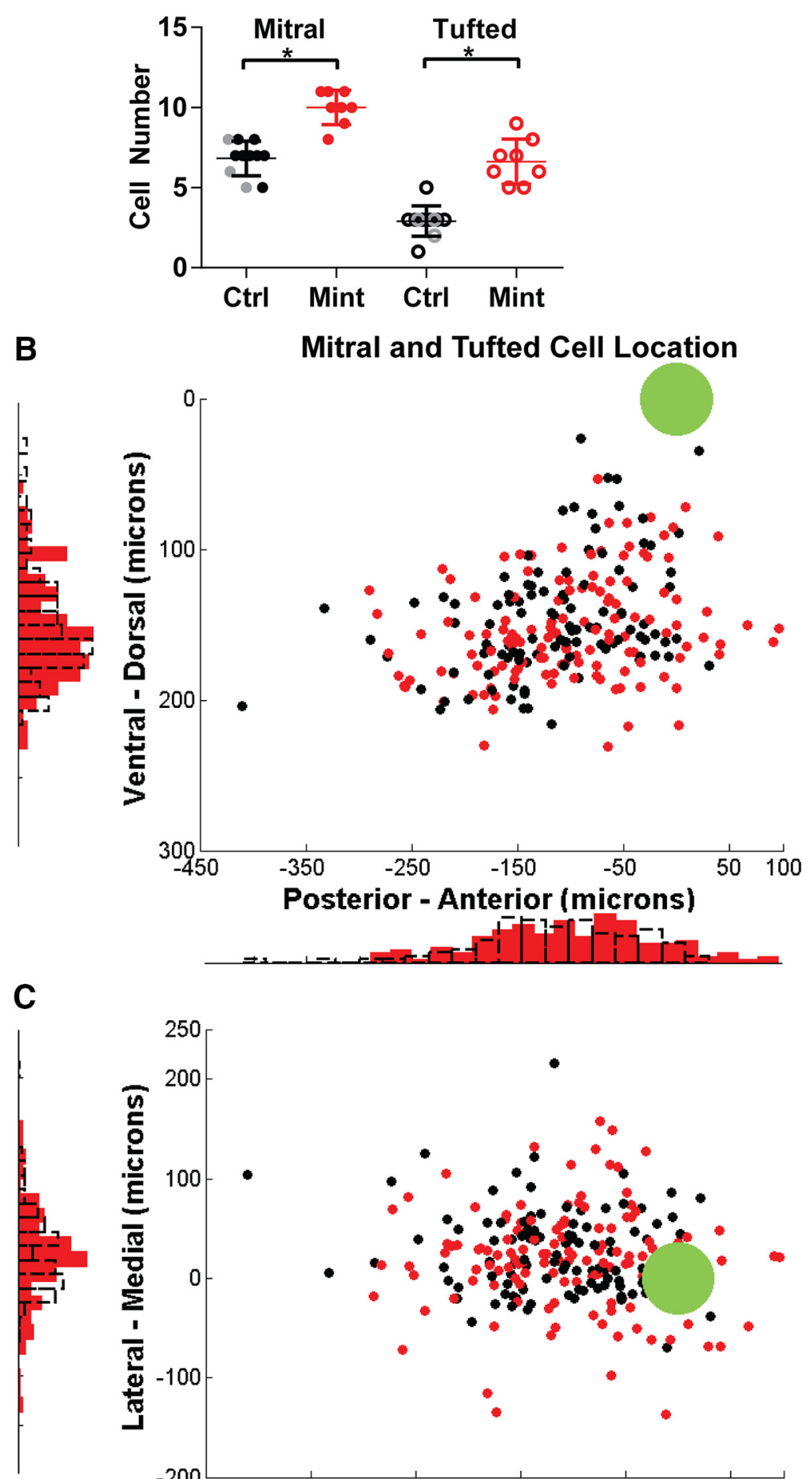

Mitral and Tufted Cells Per M72 Glomerulus

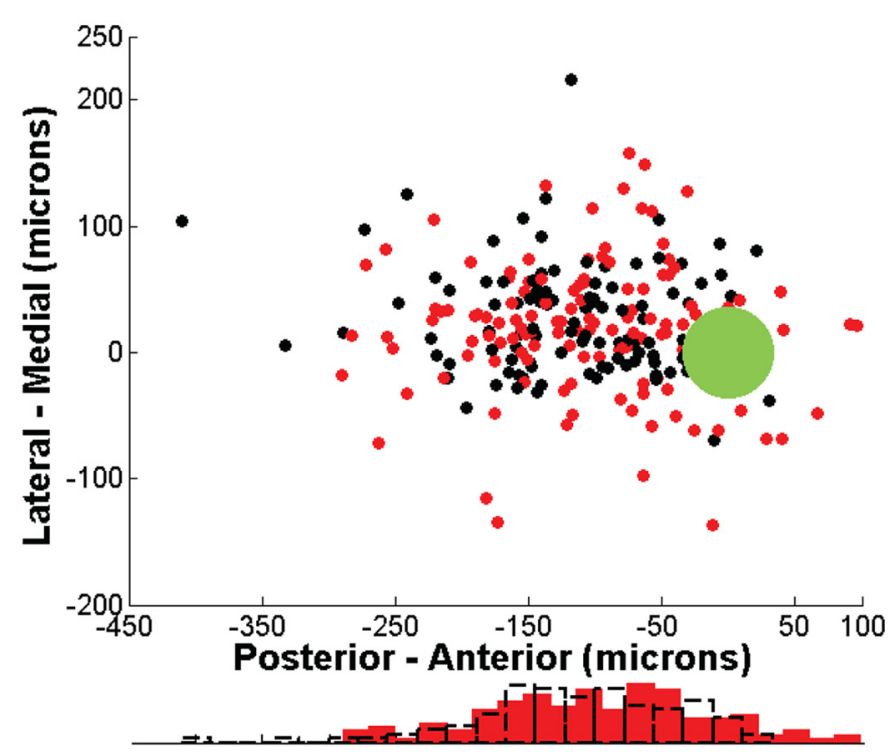

Legend - Mitral cells
O Tufted cells
Mint

Figure 2. Prenatal and early postnatal methyl salicylate exposure increases the number of M72 M/TCs. A, Number of M/TCs connected to the lateral M72 glomerulus increases after methyl salicylate exposure. Black-filled and open circles indicate electroporation of control animals with Alexa Fluor 594 hydrazide; gray-filled and open circles indicate electroporation of control animals with Alexa Fluor 594 dextran; and red-filled and open circles indicate electroporation of methyl-salicylate-exposed animals with Alexa Fluor 594 dextran. Data are shown as means \pm SD. ${ }^{*} p<0.0001$. B, C, Spatial distribution of MCs and TCs corresponding to sniffing hexanal-scented food to total time spent sniffing food: $0.23 \pm 0.25$ vs $0.65 \pm$ $0.11, n=10$ vs 9 , adjusted $p<0.0001$ ). Mint-exposed animals and hexanal-exposed animals did not demonstrate a significant difference in time spent sniffing scented food (Kruskal-Wallis test with Dunn's multiple-comparisons test; adjusted $p>0.9999$, n.s.). There was also no significant difference in odorant preference between the cohort of control animals subject to the test of mint-scented versus control food (Control $\mid \mathrm{M}$ ) and the cohort subject to the test of hexanal-scented versus control food (Control|H) (Kruskal-Wallis test with Dunn's multiple-comparisons test; adjusted $p=0.7072$, n.s.).

We established that early odorant exposure with methyl salicylate, a known M72 ligand, increases M72 glomerular volume and changes food preference. Use of a nonM72 ligand, hexanal, for odorant exposure did not increase M72 glomerular volume, but did change food preference. We next chose to investigate whether methyl salicylate odorant exposure induced additional anatomical changes to the M72 glomerular module. Specifically, we used in vivo electroporation to label and compare the population of M/TCs contained within the M72 glomerular module (Fig. 1E).

Early odorant exposure with an M72 ligand increases the number of M/TCs in the M72 glomerular module

We used in vivo electroporation to label the postsynaptic targets of the M72 glomerulus in both control M72-IRES-GFP mice and in mice that had been prenatally and postnatally exposed to methyl salicylate. This type of odorant exposure caused a remarkable increase in the number of $\mathrm{M} / \mathrm{TC}$ connected to the glomerulus (unpaired $t$ test; control vs mint; mean $\pm \mathrm{SD}$; $6.82 \pm 1.08$ vs $10.0 \pm 1.07 \mathrm{MCs} ; 2.91 \pm$ 0.94 vs $6.63 \pm 1.41$ TCs; $n=8$ vs $11, p<$ 0.0001 for both cell types), which demonstrates that early odorant exposure has a profound impact on $\mathrm{OB}$ circuit structure beyond affecting glomerulus volume (Fig. $2 A)$. The ratio of TCs to MCs within a single M72 glomerular module also increased, showing that odorant exposure increased TC number more than MC

control (black circles, 11 animals, 74 total MCs, 32 total TCs) and odor-exposed (mint) animals (red circles, 8 animals, 80 total MCs, 51 total TCs). Green circle indicates M72 glomerulus location. Histograms of cell position are next to the corresponding axes. Black-outlined bars indicate control; red solid bars indicate mint. B, Sagittal view. C, Horizontal view. Comparison of median spatial locations was nonsignificant. 
number and suggesting that chronic early odorant exposure may have differential impacts on these two parallel pathways (unpaired $t$ test; control vs mint, mean \pm SEM; TC:MC number; $0.43 \pm 0.04$ vs $0.66 \pm 0.03 ; n=11$ vs $8, p=0.0011$ ). In these mice, early odorant exposure also increased glomerulus size (data not shown) and food preference for methyl-salicylate-scented food (data pooled in with behavioral study), corroborating the findings shown in Figure 1. Age at the time of electroporation (P25P40) had no effect on measured glomerulus size.

\section{Odorant exposure does not change the spatial distribution of M/TCs postsynaptic to the M72 glomerulus}

In addition to examining the numbers of $\mathrm{M} / \mathrm{TCs}$ connected to the M72 glomerulus, we also analyzed the location of M/TC somata relative to the M72 glomerulus. An increase in M72 M/TCs distributed over a larger area in the $\mathrm{OB}$ may predict a broader range of lateral inhibition. Therefore, we measured the spatial distribution of principal neurons by plotting the locations of the labeled $\mathrm{M} / \mathrm{TCs}$ relative to the location of the M72 glomerulus (Fig. $2 B, C)$. M/TCs from control and odor-exposed groups were located in similar areas around the M72 glomerulus. All MCs from both groups were found in an area within the MCL described by an ellipse with length $450 \mu \mathrm{m}$ and width $300 \mu \mathrm{m}$. Previous measurements indicate that $\mathrm{MC}$ density in the dorsal $\mathrm{OB}$ is $\sim 56 \mathrm{MCs}$ per millimeter (Richard et al., 2010), so an ellipse of this size contains a total $\sim 345$ MCs. TCs from both groups were found at a mean distance of $126 \mu \mathrm{m}$ below the glomerular layer in an area within the EPL, with all cells located in an ellipse with length 350 $\mu \mathrm{m}$ and width $300 \mu \mathrm{m}$. There was no statistical difference between control and odor-exposed mean MC and TC location in all three coordinates (Kolmogorov-Smirnov test to compare distribution in each dimension separately resulted in a significant $p$-value of 0.045 when comparing the $y$-dimension coordinates for MCs (mean \pm SD; control vs mint; $164 \pm 21.8$ vs $170.6 \pm 23.6$ ), but a Kruskal-Wallis test with a Dunn's multiple-comparisons test yielded no significant differences between the median locations of MCs and TCs from control and odor-exposed groups. Therefore, we conclude that odorant exposure increases the number of recruited M72 M/TCs, but does not meaningfully affect their locations relative to the M72 glomerulus.

\section{In vivo electroporation reliably labels the postsynaptic targets of a single glomerulus}

In vivo electroporation has been used previously to label the complement of JGCs and principle projection neurons of a single glomerulus (Nagayama et al., 2007; Sosulski et al., 2011; He et al., 2012; Ke et al., 2013). To interpret these experiments, it is important to confirm that this approach labels cells associated with a single glomerulus reliably and specifically (Fig. 3). To test labeling consistency, we performed sequential electroporations in control M72-IRES-GFP mice $(n=7)$ with two colors of dye and using three separate electroporation protocols. Figure $3 A$ shows example physical sections (Fig. 3A1, $z$-stack images) and optical sections (Fig. 3A2) of an M72 glomerulus and labeled cells after dual-color electroporation using a stationary theta glass electrode. In this first method, two sequential electroporations were performed using a single theta glass electrode, in which a glass septum forms two isolated compartments into which two dyes (Alexa Fluor 594 dextran and Alexa Fluor 488 dextran) were back-filled, one in each compartment. A separate silver electrode wire was placed in each compartment, allowing for isolated single-color electroporations. The electrode tip position remained constant between the two electroporations. Figure $3, B$ and
$C$, shows example optical sections of M72 glomeruli from two different animals after dual-color electroporation using a repositioned theta glass electrode. In this second method, two sequential electroporations were performed, again with a single theta glass electrode and two dyes, but here we moved the electrode tip $\sim 20 \mu \mathrm{m}$ between each single-color electroporation, a substantial distance within the M72 glomerulus, which is $75-100 \mu \mathrm{m}$ at its longest diameter. Finally, Figure $3 D$ shows example optical sections of an M72 glomerulus after dual-color electroporation using two separate electrodes made from plain capillary glass, each backfilled with either Alexa Fluor 594 dextran or Alexa Fluor 488 dextran. Electroporation pulse parameters for all three approaches were kept constant, as described in the Materials and Methods section. In all of these experiments, we observed that both dyes filled the entirety of the glomerulus and identical populations of JGCs, M/TCs, and neuronal processes were labeled by both dyes, demonstrating reliable and complete labeling by the two electroporations. All dual-color electroporation experiments yielded labeled M/TC numbers within the range of labeled M/TCs reported in Figure 2. In addition, these data demonstrate that exact replication of electrode tip position within the glomerulus between electroporations is not necessary to label the same cohort of cells and cell processes. This evidence allowed us to use in vivo dye electroporation to compare numbers of labeled M/TCs connected to the M72 glomerulus between animals and odor exposure conditions.

\section{Discussion}

\section{Early odorant exposure changes the structure of a glomerular} module profoundly

Here, we demonstrate that odorant exposure during the prenatal and early postnatal period has profound effects on the composition of the glomerular module. Namely, odorant exposure with methyl salicylate, an M72 ligand, increases the number of MCs and TCs connected to the M72 glomerulus by $40 \%$ and $100 \%$, respectively.

The timing of our odorant exposure paradigm suggests that relevant early olfactory experience may specifically affect the process by which M/TC apical dendrites are pruned. Immature MCs extend multiple apical dendrites and innervate multiple nearby glomeruli (Pomeroy et al., 1990; Matsutani and Yamamoto, 2000). By P10, the majority of M/TCs complete dendritic pruning such that each M/TC projects a single apical dendrite to a single glomerulus (Malun and Brunjes, 1996; Matsutani and Yamamoto, 2000). There is some evidence that this maturation process is odor dependent (Maher et al., 2009). Our odorant exposure paradigm begins before birth and continues through early postnatal development, encompassing this refinement period, and our data show that odorant exposure induces the addition of nearby M/TCs to the M72 glomerular module (Fig. 2). Therefore, specific odorant experience may stabilize the M72glomerulus-projecting apical dendrite of M/TCs, whereas the apical dendrites projecting to other neighboring glomeruli are eliminated. This would have the secondary effect of decreasing the number of M/TCs connected to neighboring glomeruli, although this likely represents a reduction of less than one cell per nearby glomerulus.

The dense local labeling of dendrites and axons in the glomerulus generated by in vivo electroporation precludes a robust analysis of the structural changes within the glomerulus itself. The larger glomeruli produced after odorant exposure may be the product of increased OSN number or increased OSN axon 


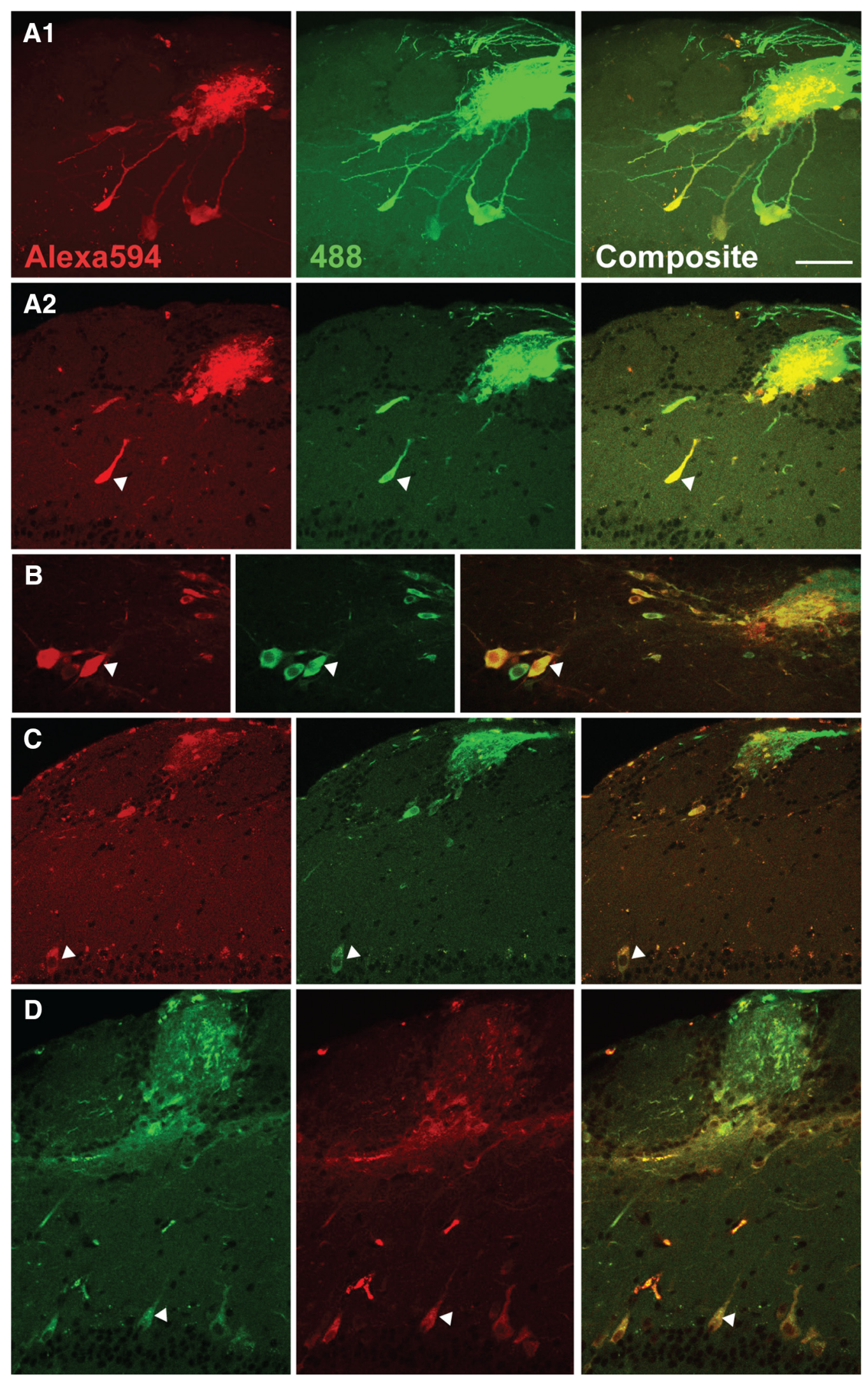

Figure 3. In vivo electroporation labels the postsynaptic targets of a single glomerulus reliably. $A$, Sequential dual-color electroporation of control M72 glomerulus using a theta glass electrode and no change in electrode tip position between sequential single-color electroporations of Alexa Fluor 594 dextran and Alexa Fluor 488 dextran. $\boldsymbol{A}$, Maximum projection image of one physical section containing the M72 glomerulus and dual-labeled JGCs and M/TCs. A2, Optical section from physical section from $\mathbf{A 1}$. White arrowhead indicates dual-labeled TC soma. B, C, 0ptical sections from separate sequential dual-color electroporations of two control M72 glomeruli using a theta glass electrode and $\sim 20 \mu \mathrm{m}$ movement of electrode tip position between single-color electroporations of Alexa Fluor 594 dextran and Alexa Fluor 488 dextran. White arrowheads indicate dual-labeled MC somata. D, Optical section from dual-color electroporation of control M72 glomerulus using two separate electrodes backfilled with either Alexa Fluor 594 dextran or Alexa Fluor 488 dextran. White arrowhead indicates dual-labeled MC. Scale bar, $50 \mu \mathrm{m}$. 
branching and odorant exposure could also increase the volume of intraglomerular processes from JGCs or M/TCs.

In this study, we examined specifically the M72 glomerulus, a dorsally located glomerulus with a number of known odorant ligands. These qualities allowed us to perform in vivo electroporation and odorant exposure with an M72 ligand. However, we believe that these results will likely generalize to other glomeruli. The M72 glomerulus has been well characterized and these analyses have been broadly applied to other glomeruli (Potter et al., 2001; Treloar et al., 2002; Feinstein and Mombaerts, 2004; Cavallin et al., 2010; Richard et al., 2010; Valle-Leija et al., 2012; Zapiec and Mombaerts, 2015). However, the relatively late development of the M72 glomerulus compared with other, more rostral glomeruli (Bailey et al., 1999; Potter et al., 2001) may mean that the effects that we observe may be more or less prominent than in glomeruli that develop earlier.

\section{Anatomical changes to the M72 glomerular module are ligand specific}

Prenatal and postnatal odorant exposure to hexanal-scented food did not increase M72 glomerular volume significantly. Because hexanal is not a high-affinity ligand for the M72 odorant receptor, these data indicate that the structural changes induced by odorant exposure are not a result of global changes across the $\mathrm{OB}$, but rather are specific to activated glomeruli. Both hexanal- and methyl-salicylate-exposed mice showed a preference for food odorized with the exposure odorant, suggesting that the lack of change in M72 glomerular volume of hexanal-exposed animals was not due to ineffective odorant exposure. Therefore, we conclude that the observed changes in glomerulus volume are activation specific. Further analysis of the M/TC network of the M72 glomerulus is necessary to investigate whether plasticity of the $\mathrm{M} / \mathrm{TC}$ network is also activation specific.

\section{Impact of increasing the number of M/TCs connected to a specific glomerulus}

Increasing the number of M/TCs connected to a single glomerulus may have several effects on odor processing. First, because $\mathrm{M} / \mathrm{TCs}$ represent the output from the $\mathrm{OB}$, increasing the cohort of cells specific to the M72 glomerular module will increase the representation of M72-glomerulus-associated information in higher cortical areas such as the piriform cortex. Second, increasing the number of M72 M/TCs may also increase the number of M72 M/TC lateral dendrites and, as a result, the number of reciprocal synapses and recurrent inhibitory synapses up to $1 \mathrm{~mm}$ away from the M72 glomerulus (Egger and Urban, 2006). Increasing the number of M72 M/TCs could increase the strength and/or number of lateral inhibitory interactions that M72 ligands evoke in other glomerular modules.

Third, our data show that odorant exposure increases the number of M72-associated M/T cells in a spatially confined region within a few hundred micrometers of the M72 glomerulus. Therefore, if we assume that all M/T cells extend lateral dendrites in a radially symmetric way (Mori et al., 1983), then this would increase the density of lateral dendrites located close to the M72 glomerulus that are activated by M72 ligands. Because these dendrites form reciprocal connections with nearby granule cells, activation of an odor-exposed M72 glomerulus would cause greater excitation of granule cells close to the M72 glomerulus, leading to increased granule-cell-mediated lateral inhibition between homotypic M72 M/TCs (Urban and Sakmann, 2002). Such homotypic lateral inhibition could also be modulated in the glomerular layer by periglomerular cells (Najac et al., 2015).

\section{Number of M/TCs associated with a single glomerular module}

Beyond the plasticity that we observed after early odorant exposure, simply knowing the number of $\mathrm{M} / \mathrm{T}$ cells for a given glomerulus is an important step toward understanding the complexity of the olfactory system. Previous work using stereological estimates to assess the number of glomeruli and MCs in the $\mathrm{OB}$ suggest that, on average, there are as many as $20-40 \mathrm{MCs} / \mathrm{glom}$ erulus in the mouse OB (Thamke et al., 1973; Benson et al., 1984; Pomeroy et al., 1990), a number and range that far exceeds any of the cell counts that we observed (maximum 11 MCs and 9 TCs). Estimates of MCs per single glomerulus also vary significantly in other species, ranging from 13-16 MCs/glomerulus in rats (Panhuber et al., 1985; Royet et al., 1998) to 9-23 MCs/glomerulus in rabbits (Allison and Warwick, 1949; Royet et al., 1998). These values were determined using estimates of the total number of glomeruli and MCs within the $\mathrm{OB}$ and therefore represent average $\mathrm{MC}$ number across all glomeruli. These estimates also vary significantly between observers, with the early mouse studies cited above reporting $\sim 40,000$ MCs and 1800 glomeruli $(\sim 21$ $\mathrm{MCs} /$ glomerulus), whereas a more recent study (Richard et al., 2010) reported 33,000 MCs and 3600 glomeruli (approximately 9 MCs/glomerulus). Sosulski et al. (2011) also reported an average of approximately $9 \mathrm{MCs} / \mathrm{glomerulus}$ after using in vivo electroporation to label the postsynaptic targets of single glomeruli located on the dorsal and lateral OB. The M/TC counts reported by both Richard et al. (2010) and Sosulski et al. (2011) are more similar to our results (average across both groups: $\sim 8 \mathrm{MCs} /$ glomerulus and $\sim 4.5 \mathrm{TCs} /$ glomerulus), suggesting that the range of M/TCs per glomerulus may be larger than previously thought. Here, we used an approach similar to that used by Sosulski et al. (2011) that allowed us to determine the number of M/TCs per glomerulus for a single, genetically identified glomerulus with low animal-to-animal variability (Fig. 3). Therefore, although our M/TC counts are lower than some of those reported previously, we believe that this may reflect the relative sparsity of M72expressing OSNs and that the specificity and reliability of our approach allow us to make valid comparisons between animals about the number of M/TCs per glomerulus. Using in vivo electroporation, we show that early odorant exposure to a ligand odorant changes the structure of both the glomerulus and the $\mathrm{M} / \mathrm{TC}$ network of the activated M72 glomerular module significantly.

\section{References}

Allison AC, Warwick RT (1949) Quantitative observations on the olfactory system of the rabbit. Brain 72:186-197. CrossRef Medline

Bailey MS, Puche AC, Shipley MT (1999) Development of the olfactory bulb: evidence for glia-neuron interactions in glomerular formation. J Comp Neurol 415:423-448. Medline

Benson TE, Ryugo DK, Hinds JW (1984) Effects of sensory deprivation on the developing mouse olfactory system: a light and electron microscopic, morphometric analysis. J Neurosci 4:638-653. Medline

Blanchart A, De Carlos JA, López-Mascaraque L (2006) Time frame of mitral cell development in the mice olfactory bulb. J Comp Neurol 496:529-543. CrossRef Medline

Cavallin MA, Powell K, Biju KC, Fadool DA (2010) State-dependent sculpting of olfactory sensory neurons attributed to sensory enrichment, odor deprivation, and aging. Neurosci Lett 483:90-95. CrossRef Medline

Dias BG, Ressler KJ (2014) Parental olfactory experience influences behavior and neural structure in subsequent generations. Nat Neurosci 17:89-96. CrossRef Medline

Egger V, Urban NN (2006) Dynamic connectivity in the mitral cell-granule cell microcircuit. Semin Cell Dev Biol 17:424-432. CrossRef Medline

Feinstein P, Mombaerts P (2004) A contextual model for axonal sorting into 
glomeruli in the mouse olfactory system. Cell 117:817-831. CrossRef Medline

He J, Tian H, Lee AC, Ma M (2012) Postnatal experience modulates functional properties of mouse olfactory sensory neurons. Eur J Neurosci 36:2452-2460. CrossRef Medline

Hovis KR, Padmanabhan K, Urban NN (2010) A simple method of in vitro electroporation allows visualization, recording, and calcium imaging of local neuronal circuits. J Neurosci Methods 191:1-10. CrossRef Medline

Ke MT, Fujimoto S, Imai T (2013) SeeDB: a simple and morphologypreserving optical clearing agent for neuronal circuit reconstruction. Nat Neurosci 16:1154-1161. CrossRef Medline

Kerr MA, Belluscio L (2006) Olfactory experience accelerates glomerular refinement in the mammalian olfactory bulb. Nat Neurosci 9:484-486. CrossRef Medline

Lin DM, Wang F, Lowe G, Gold GH, Axel R, Ngai J, Brunet L (2000) Formation of precise connections in the olfactory bulb occurs in the absence of odorantevoked neuronal activity. Neuron 26:69-80. CrossRef Medline

Maher BJ, McGinley MJ, Westbrook GL (2009) Experience-dependent maturation of the glomerular microcircuit. Proc Natl Acad Sci U S A 106: 16865-16870. CrossRef Medline

Malun D, Brunjes PC (1996) Development of olfactory glomeruli: temporal and spatial interactions between olfactory receptor axons and mitral cells in opossums and rats. J Comp Neurol 368:1-16. CrossRef Medline

Matsutani S, Yamamoto N (2000) Differentiation of mitral cell dendrites in the developing main olfactory bulbs of normal and naris-occluded rats. J Comp Neurol 418:402-410. Medline

Mombaerts P, Wang F, Dulac C, Chao SK, Nemes A, Mendelsohn M, Edmondson J, Axel R (1996) Visualizing an olfactory sensory map. Cell 87:675-686. CrossRef Medline

Mori K, Kishi K, Ojima H (1983) Distribution of dendrites of mitral, displaced mitral, tufted, and granule cells in the rabbit olfactory bulb. J Comp Neurol 219:339-355. CrossRef Medline

Nagayama S, Zeng S, Xiong W, Fletcher ML, Masurkar AV, Davis DJ, Pieribone VA, Chen WR (2007) In vivo simultaneous tracing and Ca2 + imaging of local neuronal circuits. Neuron 53:789-803. CrossRef Medline

Najac M, Sanz Diez A, Kumar A, Benito N, Charpak S, De Saint Jan D (2015) Intraglomerular lateral inhibition promotes spike timing variability in principal neurons of the olfactory bulb. J Neurosci 35:43194331. CrossRef Medline

Panhuber H, Laing DG, Willcox ME, Eagleson GK, Pittman EA (1985) The distribution of the size and number of mitral cells in the olfactory bulb of the rat. J Anat 140:297-308. Medline

Pomeroy SL, LaMantia AS, Purves D (1990) Postnatal construction of neural circuitry in the mouse olfactory bulb. J Neurosci 10:1952-1966. Medline

Potter SM, Zheng C, Koos DS, Feinstein P, Fraser SE, Mombaerts P (2001)
Structure and emergence of specific olfactory glomeruli in the mouse. J Neurosci 21:9713-9723. Medline

Richard MB, Taylor SR, Greer CA (2010) Age-induced disruption of selective olfactory bulb synaptic circuits. Proc Natl Acad Sci U S A 107:1561315618. CrossRef Medline

Royal SJ, Key B (1999) Development of P2 olfactory glomeruli in P2internal ribosome entry site-tau-LacZ transgenic mice. J Neurosci 19: 9856-9864. Medline

Royet JP, Distel H, Hudson R, Gervais R (1998) A re-estimation of the number of glomeruli and mitral cells in the olfactory bulb of rabbit. Brain Res 788:35-42. CrossRef Medline

Schaefer ML, Finger TE, Restrepo D (2001) Variability of position of the P2 glomerulus within a map of the mouse olfactory bulb. J Comp Neurol 436:351-362. Medline

Sosulski DL, Bloom ML, Cutforth T, Axel R, Datta SR (2011) Distinct representations of olfactory information in different cortical centers. Nature 472:213-216. CrossRef Medline

Strotmann J, Conzelmann S, Beck A, Feinstein P, Breer H, Mombaerts P (2000) Local permutations in the glomerular array of the mouse olfactory bulb. J Neurosci 20:6927-6938. Medline

Thamke B, Schulz E, Schönheit B (1973) Neurohistological studies on the olfactory bulb of the adult white laboratory rat (Rattus norvegicus, forma alba). J Hirnforsch 14:435-449. Medline

Todrank J, Heth G, Restrepo D (2011) Effects of in utero odorant exposure on neuroanatomical development of the olfactory bulb and odour preferences. Proc Biol Sci 278:1949-1955. CrossRef Medline

Treloar HB, Feinstein P, Mombaerts P, Greer CA (2002) Specificity of glomerular targeting by olfactory sensory axons. J Neurosci 22:2469-2477. Medline

Urban NN, Sakmann B (2002) Reciprocal intraglomerular excitation and intra- and interglomerular lateral inhibition between mouse olfactory bulb mitral cells. J Physiol 542:355-367. CrossRef Medline

Valle-Leija P, Blanco-Hernández E, Drucker-Colín R, Gutiérrez-Ospina G, Vidaltamayo R (2012) Supernumerary formation of olfactory glomeruli induced by chronic odorant exposure: a constructivist expression of neural plasticity. PLoS One 7:e35358. CrossRef Medline

Wang F, Nemes A, Mendelsohn M, Axel R (1998) Odorant receptors govern the formation of a precise topographic map. Cell 93:47-60. CrossRef Medline

Zapiec B, Mombaerts P (2015) Multiplex assessment of the positions of odorant receptor-specific glomeruli in the mouse olfactory bulb by serial two-photon tomography. Proc Natl Acad Sci U S A 112:E5873-E5882. CrossRef Medline

Zhang J, Huang G, Dewan A, Feinstein P, Bozza T (2012) Uncoupling stimulus specificity and glomerular position in the mouse olfactory system. Mol Cell Neurosci 51:79-88. CrossRef Medline 